\title{
First detailed reconstruction of the karyotype of Trachypithecus cristatus (Mammalia: Cercopithecidae)
}

\author{
Fan Xiaobo ${ }^{1}$, Krit Pinthong ${ }^{2}$, Hasmik Mkrtchyan ${ }^{3}$, Pornnarong Siripiyasing ${ }^{4}, N^{2}$ dezda Kosyakova ${ }^{1}$, \\ Weerayuth Supiwong ${ }^{5}$, Alongkoad Tanomtong ${ }^{5}$, Arunrat Chaveerach ${ }^{5}$, Thomas Liehr ${ }^{1}$, Marcelo de Bello Cioffi ${ }^{6}$ \\ and Anja Weise 1,7* $^{*}$
}

\begin{abstract}
Background: The chromosomal homologies of human (Homo sapiens = HSA) and silvered leaf monkey (Trachypithecus cristatus = TCR) have been previously studied by classical chromosome staining and by fluorescence in situ hybridization (FISH) applying chromosome-specific DNA probes of all human chromosomes in the 1980s and 1990s, respectively.

Results: However, as the resolution of these techniques is limited we used multicolor banding (MCB) at an 250-band level, and other selected human DNA probes to establish a detailed chromosomal map of TCR. Therefore it was possible to precisely determine evolutionary conserved breakpoints, orientation of segments and distribution of specific regions in TCR compared to HSA. Overall, 69 evolutionary conserved breakpoints including chromosomal segments, which failed to be resolved in previous reports, were exactly identified and characterized.

Conclusions: This work also represents the first molecular cytogenetic one characterizing a multiple sex chromosome system with a male karyotype $44, X Y_{1} Y_{2}$. The obtained results are compared to other available data for old world monkeys and drawbacks in hominoid evolution are discussed.
\end{abstract}

Keywords: Evolutionary conserved breakpoints, Multicolor banding, Old world monkeys, $X Y_{1} Y_{2}$ sex system

\section{Background}

Trachypithecus cristatus (TCR) [1], also known as silvered lutung, silvered leaf monkey or the silvery langur, belongs to superfamily Cercopithecoidea, family Cercopithecidae, subfamily Colobinae. The colobines divided during evolution into an African clade and an Asian clade [2]. TCR is widely distributed in continental Southeast Asia including Myanmar, West-central Thailand, Cambodia, Laos, Vietnam and Southern China [2-4]. Recently, four species groups were recognized (T. pileatus, T. francoisi, T. obscurus and T. cristatus), including 18 species only in the Asian colobine of Trachypithecus. Genus TCR was initially denominated with various Latin names between 1821 and 1962, like Simia cristata, Semnopithecus cristata,

\footnotetext{
* Correspondence: Anja.Weise@med.uni-jena.de

'Institute of Human Genetics, Jena University Hospital, Friedrich Schiller University, Kollegiengasse 10, Jena D-07743, Germany

${ }^{7}$ Institut für Humangenetik, Postfach, Jena D-07740, Germany

Full list of author information is available at the end of the article
}

Pygathrix cristata, Presbytis cristata, before the current name Trachypithecus cristatus was introduced [2].

The karyotype of TCR was described in 1970 as $2 n=44$ [5]. In the 1980s, chromosome banding analysis were used in TCR [6-8], including comparative R-banding of three different species of Colobus genus [6]. In 1983, G and Q banding were applied to analyze the banding patterns of a female TCR [7]. One year later, male TCR was characterized as carrying an evolutionary conserved translocation involving the $\mathrm{Y}$ chromosome and two autosomes [9]. Furthermore, since 1997 chromosomal homologies between human chromosomes and TCR has been established by fluorescence in situ hybridization (FISH) applying human whole chromosome paintings. Thus, up to now, unique reciprocal translocations corresponding to HSA Y \& 5, HSA $1 \& 19$, and HSA 6 \& 16 as well as fusions of HSA $14 \& 15$ and HSA $21 \& 22$, were characterized [10]. However, as whole chromosome paints have only a limited 
resolution [11], we established a detailed comparative chromosome map of TCR primarily based on multicolor banding $(\mathrm{MCB})$. The potential of this approach in order to clarify and to resolve evolutionary conserved chromosomal rearrangements was already shown by our group for other primates [12-14].

\section{Results}

MCB results are summarized in Figure 1 and in Tables 1 and 2. Representative results of probes specific for all acrocentric short arms in HSA, the NOR-region and
SRY are also shown in Figure 1. As outlined in Table 1 the majority of TCR chromosomes are completely homologous to one of the human chromosomes, exceptions are only TCR 5 (homologous to HSA 14 and 15), TCR 6 and 8 (homologous to HSA 1 and 19), TCR 9 and 16 (homologous to HSA 6 and 16), TCR 15 (homologous to HSA 21 and 22), and TCR Y1 and Y2 (homologous to HSA Y and 5).

The centromeric positions could be narrowed down to the subband level for all 24 TCR chromosomes (Table 1). In the following chromosomes the TCR centromeric
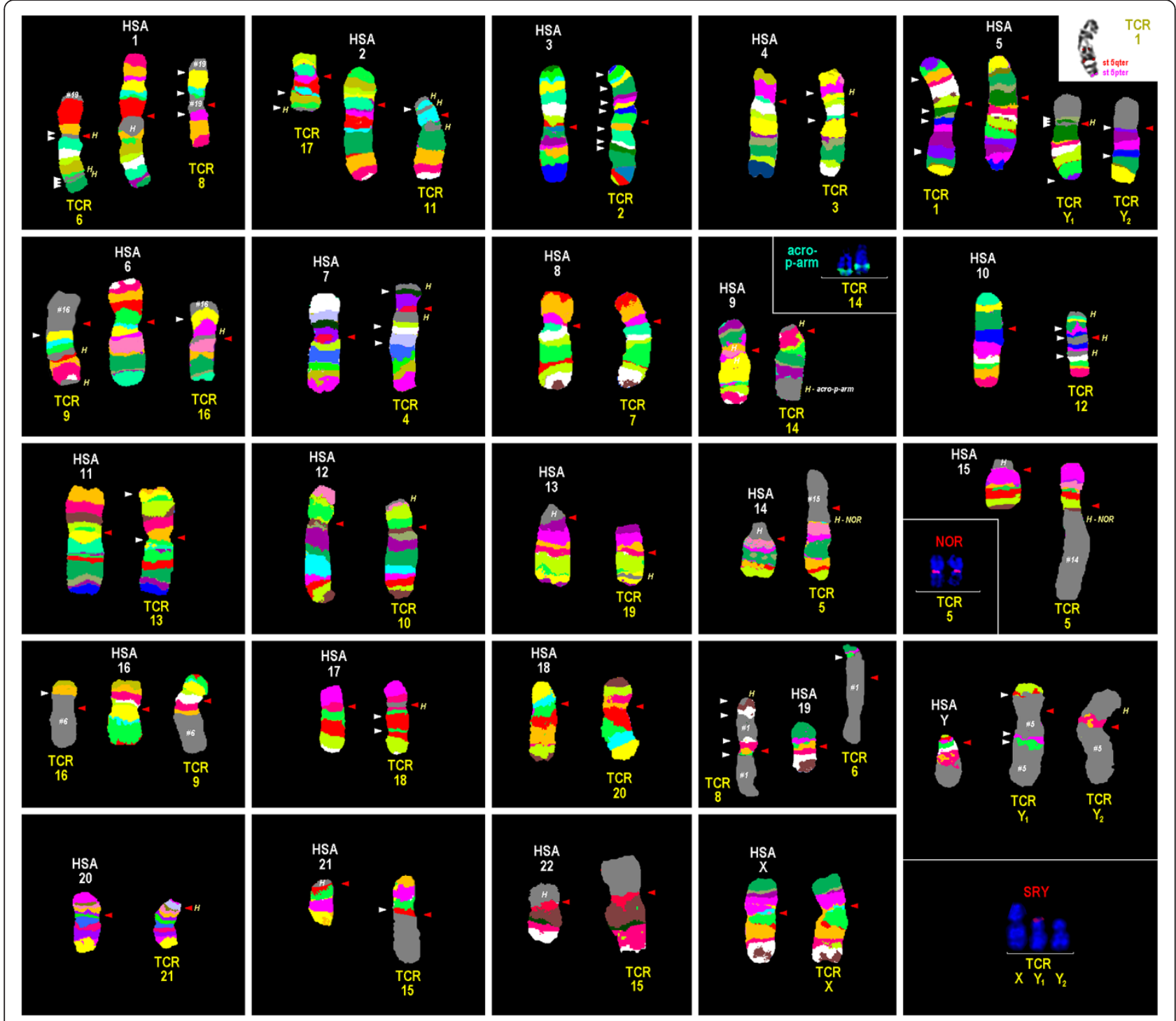

Figure 1 Representative results from this study using human MCB probes on TCR in comparison to HSA chromosomes are depicted as pseudo-colored results of HSA and TCR, at an 250-band level. HSA chromosomes are numbered by white figures, TCR chromosomes by yellow figures. The chromosomes are sorted here according to the HSA-chromosomes. In subfigures for HSA 5, 9, 14 and $Y$ there are additional FISH-results shown using probes as indicated. Red arrows stand for TCR centromeres and white arrows for HSA-centromeres. Abbreviations: \# = number of a human chromosome - region not stained by MCB probe; acro-p-arm = probe for all acrocentric short arms in HSA; $\mathrm{H}=$ heterochromatin, HSA = Homo sapiens; NOR = HSA specific FISH-probe for nucleolus organizing region; st = subtelomere; SRY = HSA specific FISH-probe for sex determining region $Y ;$ TCR = Trachypithecus cristatus. 


\begin{tabular}{|c|c|c|c|}
\hline TCR chromosome & Homologous HSA-regions (for rearrangements see Table 2) & Centromere position & Heterochromatic inserts in \\
\hline TCR 1 & HSA 5pter-5qter & like in HSA 5 & n.d.i.t.s. \\
\hline TCR 2 & HSA 3pter-3qter & HSA $3 q 26$ & n.d.i.t.s. \\
\hline TCR 3 & HSA 4pter-4qter & like in HSA 4 & n.d.i.t.s. \\
\hline \multirow[t]{2}{*}{ TCR 4} & HSA 7pter-7qter & like in HSA 7 & - end of HSA 7p \\
\hline & & & $-7 q 11.1$ \\
\hline TCR 5 & HSA 15q11.2-15qter, HSA 14q11.2-14qter & HSA $15 q 26.1 \sim 26.2$ & - fus HSA 14q11.2 / 15q26.3 \\
\hline \multirow[t]{3}{*}{ TCR 6} & HSA 1p22-1qter, HSA 19pter-19p13.2 & fus HSA $1 \mathrm{q} 22$ / 1p14 & - fus HSA 1q22 / 1q41; \\
\hline & & & - fus HSA 1q41 / 1p22; \\
\hline & & & $-1 q 24$ \\
\hline TCR 7 & HSA 8pter-8qter & like in HSA 8 & n.d.i.t.s. \\
\hline TCR 8 & HSA 1pter-1p22, HSA 19p13.2-19qter & like in HSA 19 & - end of HSA 19q \\
\hline \multirow[t]{2}{*}{ TCR 9} & HSA 6pter-6q15, HSA 16p13.1-16qter & like in HSA 16 & - end of HSA 6p; \\
\hline & & & $-6 p 21$ \\
\hline TCR 10 & HSA 12pter-12qter & like in HSA 12 & - end of HSA $12 p$ \\
\hline \multirow[t]{3}{*}{ TCR 11} & HSA 2q14.1-2qter & HSA 2q24.2 & - distal from HSA 2q14.1; \\
\hline & & & $-2 \mathrm{q} 21$ \\
\hline & & & $-2 q 31$ \\
\hline \multirow[t]{4}{*}{ TCR 12} & HSA 10pter-10qter & like in HSA 10 & - end of HSA 10p; \\
\hline & & & - fus HSA 10q21.1 / 10q22.3 \\
\hline & & & - HSA 10p11.1 \\
\hline & & & - HSA 10q11.1 \\
\hline TCR 13 & HSA 11 pter-11qter & HSA $11 \mathrm{p} 15.3$ & n.d.i.t.s. \\
\hline \multirow[t]{2}{*}{ TCR 14} & HSA 9pter-9qter & HSA 9q33 34.1 & - end of HSA 9q \\
\hline & & & - end of HSA 9p \\
\hline TCR 15 & HSA 21q11.2-21qter, HSA 22q11.21-22qter & fus $21 q 11.2 / 22 q 11.21$ & n.d.i.t.s. \\
\hline TCR 16 & HSA 6q15-6qter, 16pter-16p13.1 & HSA $6 q 21$ & - HSA 6q21 \\
\hline TCR 17 & HSA 2pter-2q14.1 & like in HSA 2 & - end of HSA $2 p$ \\
\hline TCR 18 & HSA 17pter-17qter & like in HSA 17 & - HSA 17p11.1 \\
\hline TCR 19 & HSA 13q12.1-13qter & HSA $13 q 14$ & - HSA 13q32 33 \\
\hline TCR 20 & HSA 18pter-18qter & HSA 18q21 & n.d.i.t.s. \\
\hline \multirow[t]{2}{*}{ TCR 21} & HSA 20pter-20qter & HSA 20p12 & - HSA 20p11.1 \\
\hline & & & - HSA 20q11.1 \\
\hline TCR $X$ & HSA Xpter-Xqter & like in HSA X & n.d.i.t.s. \\
\hline \multirow[t]{2}{*}{ TCR $Y_{1}$} & HSA 5p12-5q31.2, HSA Ypter-Yp11.2 & like in HSA 5 & - HSA 5p11 \\
\hline & & & - HSA 5q11.1 \\
\hline TCR $Y_{2}$ & HSA 5pter-5p12, HSA 5q31.2-5qter,HSA Yp11.2-Yq11.23 & like in HSA Y & - distal from HSA Yp11.23 \\
\hline
\end{tabular}

positions were the same as in HSA: TCR 1 ( = HSA 5), TCR 3 ( = HSA 4), TCR 4 ( = HSA 7), TCR 7 ( = HSA 8), TCR 8 ( = HSA 19), TCR $9(=$ HSA 16), TCR $10(=$ HSA 12), TCR 12 ( = HSA 10), TCR 17 ( = HSA 2), TCR 18 ( = HSA 17), TCR X ( = HSA X), TCR Y1 ( = HSA 5), and TCR Y2 ( = HSA Y). Centromere positions changed compared to HSA in TCR 2 (HSA 3q26), TCR 5
(HSA 15q26.1 26.2), TCR 6 (HSA 1q22/ 1p14), TCR 11 (HSA 2q24.2), TCR 13 (HSA 11p15.3), TCR 14 (HSA 9q33 34.1), TCR 15 (HSA 21q11.2/ 22q11.2), TCR 6 (HSA 6q21), TCR 19 (HSA 13q14), TCR 20 (HSA 18q21) and TCR 21 (HSA 20p12).

None of the aforementioned TCR centromeric regions that kept their position during evolution compared to 
Table 2 Evolutionary conserved breakpoints in TCR chromosomes compared to HSA; the positions are analyzed concerning their location in GTG-light bands, colocalization with human fragile sites and breakpoints observed in HLA and GGO using MCB-approach

\begin{tabular}{|c|c|c|c|c|c|}
\hline HSA chr. & $\begin{array}{l}\text { Evolutionary conserved breakpoints including } \\
\text { neo-centromere and heterochromatin positions }\end{array}$ & $\begin{array}{l}\text { GTG- light } \\
\text { band }\end{array}$ & $\begin{array}{l}\text { Fragile site } \\
\text { in same band }\end{array}$ & $\begin{array}{l}\text { Same breakpoint in HLA } \\
\text { [Mrasek et al., } 2003 \text { [13]] }\end{array}$ & $\begin{array}{l}\text { Same breakpoint in GGO } \\
\text { [Mrasek et al., } 2001 \text { [12]] }\end{array}$ \\
\hline \multirow[t]{4}{*}{1} & $1 p 22$ & + & FRA1D & - & - \\
\hline & $1 \mathrm{q} 22$ & - & - & - & - \\
\hline & $1 q 24$ & - & - & + & - \\
\hline & $1 q 41$ & - & FRA1R & - & - \\
\hline \multirow[t]{5}{*}{2} & $2 p 25.3$ & - & FRA2M & - & - \\
\hline & $2 q 14.1$ & - & FRA2 & - & + \\
\hline & $2 q 21$ & + & FRA2F & - & ?+ \\
\hline & $2 q 24.2$ & + & FRA2T & + & - \\
\hline & $2 q 31$ & + & FRA2G & - & - \\
\hline \multirow[t]{9}{*}{3} & $3 p 26.3$ & - & FRA3E & - & - \\
\hline & $3 p 25$ & + & FRA3F & - & - \\
\hline & $3 p 23$ & + & - & - & - \\
\hline & $3 p 21.3$ & + & FRA3H & ?+ & - \\
\hline & $3 p 14$ & - & FRA3B & ?+ & - \\
\hline & $3 q 22$ & - & FRA3N & ?+ & - \\
\hline & $3 q 25$ & + & FRA3D & + & - \\
\hline & $3 q 26$ & - & FRA3O & - & - \\
\hline & $3 q 28$ & - & FRA3P & - & - \\
\hline \multirow[t]{2}{*}{4} & $4 p 12$ & + & FRA4H & - & + \\
\hline & $4 q 22$ & - & FRA4F & - & - \\
\hline \multirow[t]{6}{*}{5} & $5 p 15.2$ & - & FRA5H & - & - \\
\hline & $5 q 11.2$ & + & FRA5I & ?+ & - \\
\hline & $5 q 21$ & - & FRA5F & - & - \\
\hline & $5 q 31.2$ & - & FRA5C & - & - \\
\hline & $5 q 35.3(a)$ & + & FRA5G & - & + \\
\hline & $5 q 35.3(b)$ & + & FRA5G & - & + \\
\hline \multirow[t]{4}{*}{6} & $6 p 25.3$ & + & - & - & - \\
\hline & $6 p 21$ & + & FRA6H & - & - \\
\hline & $6 q 15$ & + & FRA6G & + & - \\
\hline & $6 q 21$ & + & FRA6F & - & - \\
\hline \multirow[t]{4}{*}{7} & $7 p 22.3$ & + & FRA7B & + & - \\
\hline & 7p15.3 & + & - & - & - \\
\hline & $7 q 11.1$ & - & FRA7A & - & - \\
\hline & $7 q 11.23$ & + & FRA7J & - & + \\
\hline \multirow[t]{3}{*}{9} & $9 p 34.2$ & - & - & - & - \\
\hline & $9 q 24.3$ & + & - & - & - \\
\hline & $9 q 33 \sim 34.1$ & - & FRA9M & - & - \\
\hline \multirow[t]{5}{*}{10} & 10p15.3 & + & FRA10H & - & - \\
\hline & 10p11.2 & + & FRA10J & - & ?+ \\
\hline & 10p11.1 & - & - & - & - \\
\hline & $10 q 11.1$ & - & FRA10G & - & - \\
\hline & $10 q 21.1$ & - & FRA10C & + & - \\
\hline
\end{tabular}


Table 2 Evolutionary conserved breakpoints in TCR chromosomes compared to HSA; the positions are analyzed concerning their location in GTG-light bands, colocalization with human fragile sites and breakpoints observed in HLA and GGO using MCB-approach (Continued)

\begin{tabular}{|c|c|c|c|c|c|}
\hline & $10 q 22.3$ & + & FRA10D & $?+$ & + \\
\hline \multirow[t]{3}{*}{11} & $11 \mathrm{p} 15.4$ & - & - & - & - \\
\hline & $11 p 15.3$ & + & FRA11J & - & - \\
\hline & $11 q 12$ & - & - & + & - \\
\hline 12 & $12 p 13.33$ & + & FRA12F & - & - \\
\hline \multirow[t]{3}{*}{13} & $13 q 12.1$ & + & - & - & - \\
\hline & $13 q 14$ & + & FRA13G & ?+ & - \\
\hline & $13 q 32 \sim 33$ & + & FRA13D & + & - \\
\hline 14 & $14 q 11.2$ & + & FRA14D & ?+ & + \\
\hline \multirow[t]{2}{*}{15} & $15 q 11.2$ & + & FRA15C & ?+ & - \\
\hline & $15 q 26.1 \sim 26.2$ & + & FRA15G & ?+ & - \\
\hline 16 & 16p13.1 & + & FRA16H & + & - \\
\hline \multirow[t]{3}{*}{17} & 17p11.1 & - & FRA17C & ?+ & - \\
\hline & $17 q 21.3$ & + & FRA17D & ?+ & - \\
\hline & $17 q 24$ & - & FRA17E & + & - \\
\hline 18 & $18 q 21$ & + & FRA18B & - & - \\
\hline \multirow[t]{3}{*}{19} & 19p13.2 & - & - & ?+ & - \\
\hline & $19 q 13.2$ & - & - & + & - \\
\hline & $19 q 13.43$ & - & - & ?+ & - \\
\hline \multirow[t]{3}{*}{20} & $20 p 12$ & - & FRA20B & - & - \\
\hline & 20p11.1 & - & - & - & - \\
\hline & $20 q 11.1$ & - & FRA20D & - & - \\
\hline 21 & $21 q 11.2$ & + & FRA21 & ?+ & - \\
\hline 22 & $22 q 11.21$ & + & - & ?+ & - \\
\hline \multirow[t]{3}{*}{ Y } & Yp11.31 & - & - & - & - \\
\hline & Yp11.2 & + & - & - & - \\
\hline & Yq11.23 & + & - & - & - \\
\hline
\end{tabular}

Abbreviations: - = no; + = yes; ? + = most likely yes; $\mathrm{a}$ and b in 5q35.3 = break within subtelomere region.

human showed positive FISH-signals with any of the used HSA centromere specific probes (data not shown). Also, none of the other human heterochromatin specific probes from HCM probe set gave any specific signals in TCR, with 2 exceptions: NOR-specific signals were observed in TCR 5 (at fusion of HSA 14q11.2 and 15q26.3) (Figure 1) and the probes midi54 (Figure 1) and midi 36 (data not shown); the latter two located on the distal end of the long arm of TCR 14.

In the literature there were 81 TCR specific heterochromatic insertions and/ or additions to chromosomes reported (Figure 2). In this study only 25 of them were confirmed and mapped (Figure 2 and Table 1, last column).

Table 2 summarizes the 69 evolutionary conserved breakpoints observed in TCR in this study; they are given according to the homologous regions in HSA. Only HSA chromosomes X (TCR X) and 8 (TCR 7) are completely unaltered during evolution from a common ancestor of HSA and TCR. All other homologous of TCR chromosomes have undergone one (HSA 12, 14, 16, 18, 21 and 22), two (HSA 4 and 15), three (HSA Y, 11, 13, 17, 19, and 20), four (HSA 1 and 6), five (HSA 2), six (HSA 5, and 10), or nine (HSA 3) evolutionary conserved break events during speciation in respect to the human karyotype.

Besides, the characterized breakpoints of TCR are compared with such previously mapped in Hylobates lar (HLA) and Gorilla gorilla (GGO) using MCB approach (Table 2). Again, an alignment of the breakpoint and their positioning in GTG-light bands and their spatial proximity to human fragile sites was done.

\section{Discussion}

The present study represents the first one that comprehensively characterizes the karyotype of TCR. In general, 


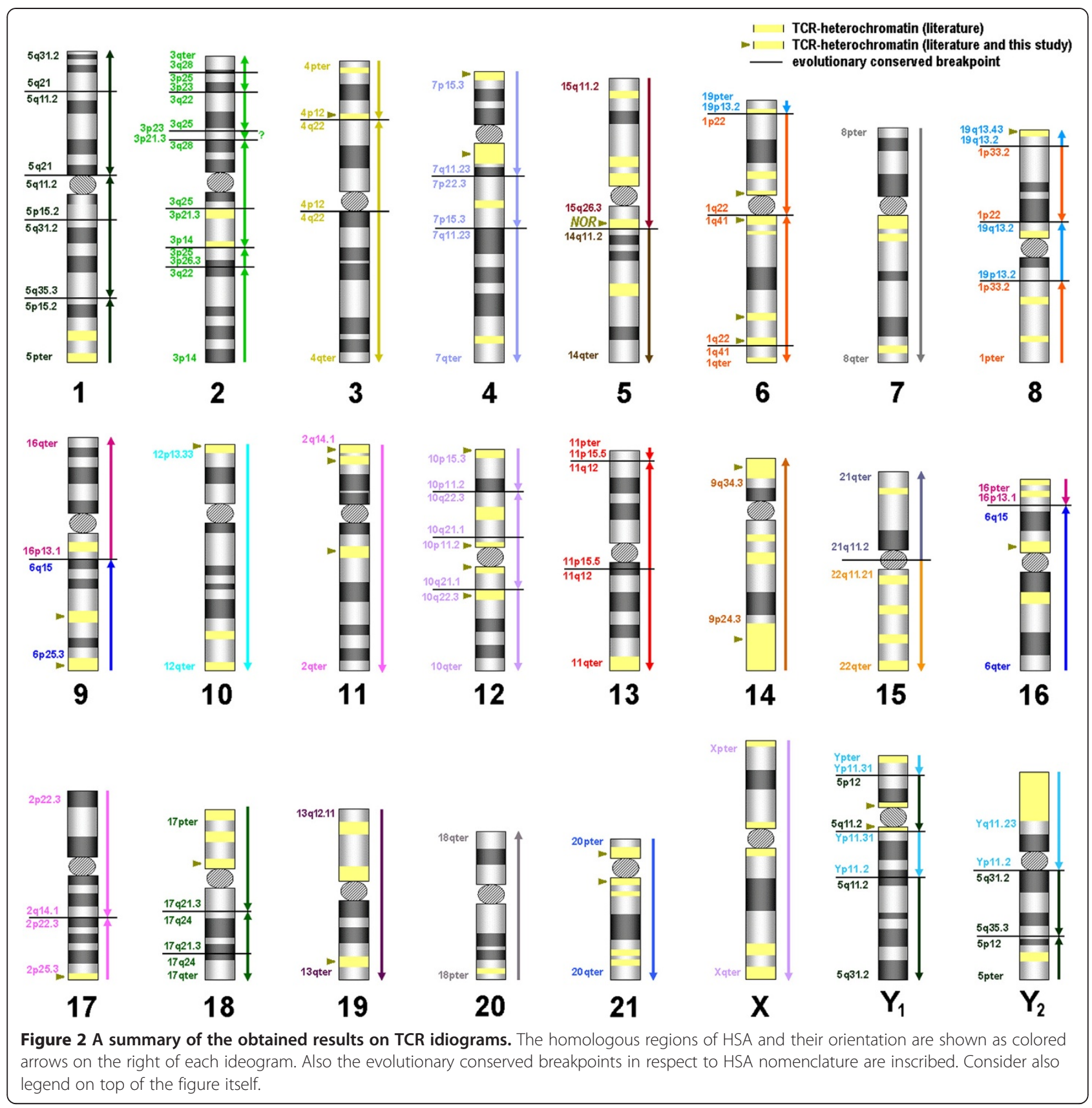

previous homologies of HSA and TCR chromosomes could be confirmed [10]. However, in this study, homologous regions for TCR chromosomes 4, 10, 11, 14, 17, 18 and 21 (that were not studied before) [10] were specifically aligned to their HSA-homologous. In contrast to [10] NOR was mapped here to the fusion points of HSA 14 and HSA 15, i.e. TCR 5 and not TCR 15. In our two studied individuals derived from Thailand, no differences in TCR 1 banding pattern were seen, which is in accordance with the literature [10].

For the first time, the exact breakpoints could be determined for the extremely rearranged karyotype of TCR, in comparison to HSA. In fact, 69 evolutionary conserved breakpoints were determined in a male TCR and confirmed in a female individual, excluding $\mathrm{Y}_{1}$ and $\mathrm{Y}_{2}$ chromosomes, obviously.

In this study no special attention was given to the centromeric regions of TCR, i.e. they were not detailed characterized as in other studies e.g. by [15] or [16]. However, a first impression is provided in which centromeres kept their positions during evolution from common ancestors to HSA and TCR and were neo-centromeres (Table 1), i.e. $\sim 50 \%$ of them stayed at the same positions and $\sim 50 \%$ moved either in one of the two species or in 
both. As expected from the literature [17], even the centromeric regions that kept their positions did not have the identical alphoid sequences in HSA and TCR.

Previous studies in human chromosomal rearrangements revealed that the majority of them (70-88\%) are found in G-light sub-bands [18]. In contrast only 37 (45.5\%) of the 69 evolutionary conserved breakpoints of TCR were located in GTG-light bands (Table 2). However, 70\% of the here observed TCR breakpoints colocalized with human fragile sites [19] supporting their potential role in the "Fragile Breakage Model" [20] and in the formation of evolutionary chromosomal rearrangements [21-26] (Table 2).

Concerning evolution it is interesting to report that in TCR and in HLA 11 evolutionary conserved breakpoints are identical and 15 more are most likely in concordance to each other. Even more interesting, 6 identical and 2 most likely identical evolutionary conserved breakpoints were identified in TCR and in GGO (Table 2). These findings need to be confirmed in further studies by locusspecific probes, and if confirmed, they will be very useful for the reconstruction of a common ancestral karyotype. Compared to the postulated Hominidea ancestral karyotype proposed by [25], only four chromosomes remained unchanged in TCR, i.e. chromosomes 4, 7, 11 and X, eleven chromosomes underwent only intrachromosomal changes like inversions (TCR 2, 3, 10, 12, 13, 14, 17, 18, 19, 20, 21) and two TCR chromosomes resulted from a fusion of ancestral chromosomes (TCR 5 and 15).

Interestingly, the regions between TCR 1 and TCR $\mathrm{Y}_{1}$ and TCR $Y_{2}$ being homologous to HSA 5 were shown to be subject to different evolutionary conserved rearrangements. Broadly speaking, TCR Y1 is homologous to TCR $1 \mathrm{p}$ and TCR Y2 to TCR 1q. However, each arm of chromosome TCR 1 underwent a further paracentric inversion, most likely being important to separate the sex chromosomes from the chromosome 1 during meiosis. Thus, a $X Y_{1} Y_{2}$ sex chromosome system is present in $T C R$, and not an $X_{1} X_{2} Y_{1} Y_{2}$ system as initially suggested [10]. However, as in TCR from Indonesia, two other forms of TCR 1 chromosome could be found [10]. Therefore, the existence of an $\mathrm{X}_{1} \mathrm{X}_{2} \mathrm{Y}_{1} \mathrm{Y}_{2}$ system cannot be completely excluded by this study.

The sex determination system in mammals is usually highly conserved as XY-system. However, multiple sex chromosome systems, like the one present in TCR and few other apes $[27,28]$ are exceptionally found in some species of e.g. the orders Insectivora, Chiroptera, Artiodactyla, Rodentia [29], and in marsupials [30]. In general, constitutional Y-chromosome / autosome translocations in human appear de novo and have a deleterious effect and, although the infertility is the only common feature, other clinical symptoms can also be observed depending on the involved breakpoints [31]. In such cases, the infertility is thought to be a result of disruption of the sex vesicle during meiosis
[32]. From this point of view it is hard to imagine conditions which are in favor of developing a multiple sex- from an XY-chromosome system. On the other hand, in population genetic models of Y-autosome and X-autosome rearrangements the population can gain a selective advantage under a wide range of conditions. If they can invade the population, Y-autosome rearrangements always spread to fixation, whereas $\mathrm{X}$-autosome rearrangements may be maintained as stable polymorphisms" [33]. The $\mathrm{XY}_{1} \mathrm{Y}_{2}$ sex chromosome system observed in TCR fits well into the suggestions of [34] that (i) female meiotic drive is the major contribution to the evolution of neo-sex chromosomes and (ii) that "in mammals, the $\mathrm{XY}_{1} \mathrm{Y}_{2}$ sex chromosome system is more prevalent in species with karyotypes of more biarmed chromosomes" rather than in species with acrocentric chromosomes. Research on meiotic behavior of such sex systems is scarce; however, one study on Bolivian owl monkey (Aotus spec.) showed that no XY pairing was observable but the Y-chromosomes formed trivalents with an autosome during gametogenesis [27].

\section{Conclusions}

In conclusion, the presented comparative map of TCR karyotype gives new insights into primate evolution and can be used as a starting point for further detailed analyses. Evolutionary conserved breakpoints, TCR-specific heterochromatic regions, centromeric sequences as well as the sex chromosome system can be fruitful fields of research in near future.

\section{Methods}

\section{Cell culture and chromosomal preparation}

Immortalized lymphoblast cell lines derived from a male and female TCR, were provided by the Khon Kaen University, Thailand. Culture techniques and chromosome preparation followed standard protocols.

\section{Fluorescence in situ hybridization}

General considerations for MCB labeling schemes and details regarding probe preparation and labeling have been described before [12,35,36]. Single and dual-color FISH techniques were performed for the applied bacterial artificial chromosome (BAC-) probes [37]. Locus-specific BAC clones were purchased at BAC/PAC Chori and DNA was isolated, PCR-amplified and labeled as described before [37]; and probes RP11-475I16 and RP11-395 L14 (both in 2q14.1), RP11-110A24 (in 19p13.3), RP-11457 M7 (in Yp11.2) and RP11-122 L9 (in Yp11.31) were applied in this study. Besides, commercially available human derived probes for the SRY gene on the Y-chromosome, HSA probes for subtelomeric regions 3pter, 3qter, 5pter, 5qter, 6pter, 6qter, 7pter, 7qter, 9pter, 9qter, 10pter, 10qter, 18pter, 18qter, 19pter, 19qter, XYpter and XYqter, and centromeric probes cep 2, cep4, cep 7, сер 8, сер 10, сер 12, сер 16, сер 
17, cep X cep Y (Abbott/ Vysis, Wiesbaden, Germany) and SE 1/5/19, SE 13/21 and SE 14/22 (Kreatech, Amsterdam, The Netherlands) were also applied.

Additionally, the following homemade HSA derived microdissection probes were used: a probe specific for the short arm of all human acrocentric chromosomes (midi54) [12,13], and partial chromosome paints for $3 p$, 3q, 5p, 5q, 6p, 6q, 7p, 7q, 9p, 9q, 10p, 10q, 18p, 18q, 19p, 19q, Yp and Yq [35]. Furthermore, the heterochromatin mix (HCM-) probe set [38] covering chromosomal regions 1q12, 16q11.2, 9q12, 9p12/ 9q13 (midi36) 15p11.2-p11.1, 19p12/q12 and Yq12 and subcentromere specific multicolor FISH (subcen-FISH) for chromosomes 3, 6, 7, 9, 11, 13 and 20 were also applied [36].

\section{Microscopic evaluation}

Images were captured using an Axioplan II microscope (Carl Zeiss Jena GmbH, Germany) equipped with filter sets for DAPI, FITC, TR, SO, Cy5 and DEAC. Image analysis was done using pseudocolor banding and fluorochrome profiles of the ISIS digital FISH imaging system (Meta Systems Hard \& Software GmbH, Altlussheim, Germany). At least 10 up to 20 metaphases were recorded, derived from a male and a female TCR for each applied probe and probe set.

\section{Competing interests}

Authors declare that there is no conflict of interest.

\section{Authors' contributions}

XF carried out molecular cytogenetic studies and the draft of the manuscript, KP carried out the molecular cytogenetic studies, HM carried out the molecular cytogenetic studies, PS carried out the molecular cytogenetic studies, NK carried out the molecular cytogenetic studies, WS carried out the cell culture, AT carried out the cell culture, AC carried out the cell culture and participated in manuscript discussion, TL participated in the design of the study and the manuscript, MBC participated in the design of the study and the manuscript discussion, AW participated in the design and coordinated the study and the manuscript. All authors read and approved the final manuscript.

\section{Acknowledgments}

This work was supported by the China Scholarship Council.

\begin{abstract}
Author details
'Institute of Human Genetics, Jena University Hospital, Friedrich Schiller University, Kollegiengasse 10, Jena D-07743, Germany. ${ }^{2}$ Faculty of Science and Technology, Surindra Rajabhat University, 186 Moo 1, Surin, Maung District 32000, Thailand. ${ }^{3}$ Center of Medical Genetics and Primary Health Care, Abovyan Str 34/3, 001, Yerevan, Armenia. ${ }^{4}$ Faculty of Science and Technology, Rajabhat Maha Sarakham University, 80 Nakonsawan Rd, Maha Sarakham, Talad, Maung District 44000, Thailand. ${ }^{5}$ Department of Biology, Faculty of Science, Khon Kaen University, 123 Moo 16 Mittapap Rd., Khon Kaen, Muang District 40002, Thailand. 'Departamento de Genética e Evolução, Universidade Federal de São Carlos, São Carlos, SP, Brazil. ${ }^{7}$ Institut für Humangenetik, Postfach, Jena D-07740, Germany.
\end{abstract}

Received: 15 November 2013 Accepted: 21 November 2013 Published: 17 December 2013

\section{References}

1. Raffles TS: Descriptive catalogue of a zoological collection, made on account of the Honourable East India Company, in the island of
Sumatra and its vicinity, under the direction of Sir Thomas Stanford Raffles, Lieutenant-Governor of Fort Marlborough: with additional notices of the natural history of those countries. Transact Linn Soc London 1821, 13:239-274.

2. Harding LE: Trachypithecus cristatus (Primates: Cercopithecidae). Mamm Species 2010, 42:149-165.

3. Fooden J: Primates obtained in peninsular Thailand June-July, 1973, with notes on the distribution of continental Southeast Asian leaf- monkeys (Presbytis). Primates 1976, 17:95-118.

4. Roos C, Nadler T, Walter L: Mitochondrial phylogeny, taxonomy and biogeography of the silvered langur species group (Trachypithecus cristatus). Mol Phylogenet Evol 2008, 47:629-636.

5. Hsu TC, Benirschke K: In An atlas of mammalian chromosomes. Edited by Hsu TC, Benirschke K. NewYork: Springer-Verlag, Folio 199; 1970.

6. Dutrillaux B, Couturier J, Muleris M, Lombard M, Chauvier G: Chromosomal phylogeny of forty-two species or subspecies of cercopithecoids (Primates Catarrhini). Ann Genet 1982, 25:96-109.

7. Ponsa M, de Boer LEM, Egozcue J: Banding patterns of the chromosomes of Presbytis cristatus pyrrhus and P. obscurus. Am J Primatol 1983, 4:165-169.

8. Muleris M, Couturier J, Dutrillaux B: Phylogénie chromosomique des Cercopithecoidea. Mammalia 1986, 50:38-52.

9. Dutrillaux B, Webb G, Muleris M, Couturier J, Butler R: Chromosome study of Presbytis cristatus: presence of a complex Y-autosome rearrangement in the male. Ann Genet 1984, 27:148-153.

10. Bigoni F, Koehler U, Stanyon R, Ishida T, Wienberg J: Fluorescene in situ hybridization establishes homology between human and silvered leaf monkey chromosomes, reveals reciprocal translocations between chromosomes homologous to human $Y / 5,1 / 9$, and $6 / 16$, and delineates an X1X2Y1Y2/X1X1X2X2 sex-chromosome system. Am J Phys Anthropol $1997,102: 315-327$.

11. Graphodatsky AS, Trifonov VA, Stanyon R: The genome diversity and karyotype evolution of mammals. Mol Cytogenet 2011, 4:22.

12. Mrasek K, Heller A, Rubtsov N, Trifonov V, Starke H, Rocchi M, Claussen U, Liehr T: Reconstruction of the female Gorilla gorilla karyotype using 25-color FISH and multicolor banding (MCB). Cytogenet Cell Genet 2001, 93:242-248.

13. Mrasek K, Heller A, Rubtsov N, Trifonov V, Starke H, Claussen U, Liehr T: Detailed Hylobates lar karyotype defined by 25 -color FISH and multicolor banding. Int J Mol Med 2003, 12:139-146.

14. Weise A, Heller A, Starke H, Mrasek K, Kuechler A, Pool-Zobel BL, Claussen U, Liehr T: Multitude multicolor chromosome banding (mMCB) - a comprehensive one-step multicolor FISH banding method. Cytogenet Genome Res 2003, 103:34-39.

15. Stanyon R, Rocchi M, Capozzi O, Roberto R, Misceo D, Ventura M, Cardone MF, Bigoni F, Archidiacono N: Primate chromosome evolution: ancestral karyotypes, marker order and neocentromeres. Chromosome Res 2008, 16:17-39.

16. Ventura M, Antonacci F, Cardone MF, Stanyon R, D'Addabbo P, Cellamare A, Sprague LJ, Eichler EE, Archidiacono N, Rocchi M: Evolutionary formation of new centromeres in macaque. Science 2007, 316:243-246.

17. Rocchi M, Archidiacono N, Schempp W, Capozzi O, Stanyon R: Centromere repositioning in mammals. Heredity 2012, 108:59-67.

18. Manvelyan M, Schreyer I, Höls-Herpertz I, Köhler S, Niemann R, Hehr U, Belitz B, Bartels I, Götz J, Huhle D, Kossakiewicz M, Tittelbach H, Neubauer S, Polityko A, Mazauric ML, Wegner R, Stumm M, Küpferling P, Süss F, Kunze H, Weise A, Liehr T, Mrasek K: Forty-eight new cases with infertility due to balanced chromosomal rearrangements: detailed molecular cytogenetic analysis of the 90 involved breakpoints. Int J Mol Med 2007, 19:855-864.

19. Mrasek K, Schoder C, Teichmann AC, Behr K, Franze B, Wilhelm K, Blaurock $\mathrm{N}$, Claussen U, Liehr T, Weise A: Global screening and extended nomenclature for 230 aphidicolin-inducible fragile sites, including 61 yet unreported ones. Int J Oncol 2010, 36:929-940.

20. Pevzner P, Tesler G: Human and mouse genomic sequences reveal extensive breakpoint reuse in mammalian evolution. Proc Natl Acad Sci U S A 2003, 100:7672-7677.

21. Bailey JA, Baertsch R, Kent WJ, Haussler D, Eichler EE: Hotspots of mammalian chromosomal evolution. Genome Biol 2004, 5:R23.

22. Murphy WJ, Larkin DM, Everts-van der Wind A, Bourque G, Tesler G, Auvil L, Beever JE, Chowdhary BP, Galibert F, Gatzke L, Hitte C, Meyers SN, Milan D, Ostrander EA, Pape G, Parker HG, Raudsepp T, Rogatcheva MB, Schook LB, Skow LC, Welge M, Womack JE, O'brien SJ, Pevzner PA, Lewin HA: 
Dynamics of mammalian chromosome evolution inferred from multispecies comparative maps. Science 2005, 309:613-617.

23. Ruiz-Herrera A, Castresana J, Robinson TJ: Is mammalian chromosomal evolution driven by regions of genome fragility? Genome Biol 2006, 7:R115.

24. Ruiz-Herrera A, Robinson TJ: Chromosomal instability in Afrotheria: fragile sites, evolutionary breakpoints and phylogenetic inference from genome sequence assemblies. BMC Evol Biol 2007, 7:199.

25. Misceo D, Capozzi O, Roberto R, Dell'oglio MP, Rocchi M, Stanyon R, Archidiacono N: Tracking the complex flow of chromosome rearrangements from the Hominoidea Ancestor to extant Hylobates and Nomascus Gibbons by high-resolution synteny mapping. Genome Res 2008, 18:1530-1537.

26. Alekseyev MA, Pevzner PA: Comparative genomics reveals birth and death of fragile regions in mammalian evolution. Genome Biol 2010, 11:R117.

27. Ma NS, Elliott MW, Morgan L, Miller A, Jones TC: Translocation of Y chromosome to an autosome in the Bolivian owl monkey, Aotus. Am J Phys Anthropol 1976, 45:191-202.

28. Solari AJ, Rahn MI: Fine structure and meiotic behaviour of the male multiple sex chromosomes in the genus Alouatta. Cytogenet Genome Res 2005, 108:262-267.

29. Fredga K: Unusual sex chromosome inheritance in mammals. Philos Trans R Soc Lond B Biol Sci 1970, 259:15-36.

30. Toder R, O'Neill RJ, Wienberg J, O'Brien PC, Voullaire L, Marshall-Graves JA: Comparative chromosome painting between two marsupials: origins of an XX/XY1Y2 sex chromosome system. Mamm Genome 1997, 8:418-422.

31. Conte RA, Kleyman SM, Klein V, Bialer MG, Verma RS: Characterization of a de novo $t(Y ; 9)$ (q11.2;q22) by FISH technique. Ann Genet 1996, 39:10-15.

32. Pinho MJ, Neves R, Costa P, Ferrás C, Sousa M, Alves C, Almeida C, Fernandes S, Silva J, Ferrás L, Barros A: Unique t(Y;1)(q12;q12) reciprocal translocation with loss of the heterochromatic region of chromosome 1 in a male with azoospermia due to meiotic arrest: a case report. Hum Reprod 2005, 20:689-696.

33. Charlesworth B, Wall JD: Inbreeding, heterozygote advantage and the evolution of neo-X and neo-Y sex chromosomes. Proceedings: Biological Sciences 1999, 266:51-56.

34. Yoshida K, Kitano J: The contribution of female meiotic drive to the evolution of neo-sex chromosomes. Evolution 2012, 66:3198-3208.

35. Liehr T, Claussen U: Current developments in human molecular cytogenetic techniques. Curr Mol Med 2002, 2:283-297.

36. Weise A, Mrasek K, Fickelscher I, Claussen U, Cheung SW, Cai WW, Liehr T, Kosyakova N: Molecular definition of high-resolution multicolor banding probes: first within the human DNA sequence anchored FISH banding probe set. J Histochem Cytochem 2008, 56:487-493.

37. Liehr T, Heller A, Starke H, Rubtsov N, Trifonov V, Mrasek K, Weise A, Kuechler A, Claussen U: Microdissection based high resolution multicolor banding for all 24 human chromosomes. Int J Mol Med 2002, 9:335-339.

38. Bucksch M, Ziegler M, Kosayakova N, Mulatinho MV, Llerena JC Jr, Morlot S, Fischer W, Polityko AD, Kulpanovich Al, Petersen MB, Belitz B, Trifonov V, Weise A, Liehr T, Hamid AB: A new multicolor fluorescence in situ hybridization probe set directed against human heterochromatin: HCM-FISH. J Histochem Cytochem 2012, 60:530-536.

\section{Submit your next manuscript to BioMed Central and take full advantage of:}

- Convenient online submission

- Thorough peer review

- No space constraints or color figure charges

- Immediate publication on acceptance

- Inclusion in PubMed, CAS, Scopus and Google Scholar

- Research which is freely available for redistribution

Submit your manuscript at www.biomedcentral.com/submit
Ciomed Central 